\title{
Epigenetic Regulation by Agonist-Specific Aryl Hydrocarbon Receptor Recruitment of Metastasis-Associated Protein 2 Selectively Induces Stanniocalcin 2 Expression $\$$
}

\author{
Aditya D. Joshi, Ekram Hossain, and Cornelis J. Elferink \\ Department of Pharmacology and Toxicology (A.D.J., C.J.E.) and Sealy Center for Environmental Health and Medicine (E.H., C.J.E.), \\ University of Texas Medical Branch, Galveston, Texas
}

Received March 20, 2017; accepted June 28, 2017

\begin{abstract}
The aryl hydrocarbon receptor (AhR) is a ligand-activated transcription factor that regulates a plethora of target genes. Historically, the AhR has been studied as a regulator of xenobiotic metabolizing enzyme genes, notably cytochrome P4501A1 encoded by CYP1A1, in response to the exogenous prototypical ligand $2,3,7,8$ tetrachlorodibenzo- $p$-dioxin (TCDD). AhR activity depends on its binding to the xenobiotic response element (XRE) in partnership with the AhR nuclear translocator (Arnt). Recent studies identified stanniocalcin 2 (Stc2) as a novel AhR target gene responsive to the endogenous $A h R$ agonist cinnabarinic acid (CA). CA-dependent AhR-XRE-mediated Stc2 upregulation is responsible for cytoprotection against ectoplasmic reticulum/ oxidative stress-induced apoptosis both in vitro and in vivo. Significantly, CA but not TCDD induces expression of Stc2 in
\end{abstract}

hepatocytes. In contrast to TCDD, CA is unable to induce the CYP1A1 gene, thus revealing an AhR agonist-specific mutually exclusive dichotomous transcriptional response. Studies reported here provide a mechanistic explanation for this differential response by identifying an interaction between the AhR and the metastasis-associated protein 2 (MTA2). Moreover, the AhR-MTA2 interaction is CA-dependent and results in MTA2 recruitment to the Stc2 promoter, concomitant with agonist-specific epigenetic modifications targeting histone $\mathrm{H} 4$ lysine acetylation. The results demonstrate that histone $\mathrm{H} 4$ acetylation is absolutely dependent on CA-induced AhR and MTA2 recruitment to the Stc2 regulatory region and induced Stc2 gene expression, which in turn confers cytoprotection to liver cells exposed to chemical insults.

\section{Introduction}

The aryl hydrocarbon receptor (AhR) is a ubiquitous, ligandactivated basic helix-loop-helix transcription factor extensively characterized in the context of 2,3,7,8-tetrachlorodibenzo- $p$ dioxin (TCDD) toxicity, as reviewed by White and Birnbaum (2009). Upon ligand binding, the deduced canonical signaling mechanism involves translocation of the cytosolic chaperonebound AhR into the nucleus, followed by dimerization with the aryl hydrocarbon receptor nuclear translocator (Arnt) protein and binding of the AhR-Arnt complex to cis-acting xenobiotic response elements (XRE) associated with target genes to regulate gene expression (Beischlag et al., 2008). Furthermore, research has revealed that the AhR is responsive to a diverse array of ligands (Denison et al., 2011; Soshilov and Denison, 2014) and that AhR functionality can be

This work was supported by the National Institutes of Health National Institute of Diabetes and Digestive and Kidney Diseases [Grant K01DK102514] and National Institute of Environmental Health Sciences [Grants R01ES026874, R21ES024607, P30ES006676].

https://doi.org/10.1124/mol.117.108878.

S This article has supplemental material available at molpharm.aspetjournals.org. differentially altered by the specific ligand binding, resulting in distinct transcriptomic responses (Ovando et al., 2010; Hrubá et al., 2011; Goodale et al., 2013). Although the precise mechanism for the differential responsiveness remains unresolved, the possibility that ligand-dependent effects may be attributed to recruitment of distinct coactivators has been proposed (Zhang et al., 2008).

In addition to the many exogenous AhR ligands identified to date, the repertoire of $\mathrm{AhR}$ agonists recently increased to include several endogenous compounds identified as tryptophan catabolites (Rannug et al., 1995; Wei et al., 1998; Opitz et al., 2011; Lowe et al., 2014). These include kynurenine, 6-formylindolo [3,2-b] carbazole, and cinnabarinic acid (CA). Using CA, we recently detected stanniocalcin 2 (Stc2) as a novel AhR target gene (Joshi et al., 2015a). Strikingly, Stc2 was completely unresponsive to induction by the exogenous AhR agonists TCDD, 3-methylchloranthrene, or $\beta$-naphthoflavone (Harper et al., 2012). Conversely, CYP1A1 gene induction was unresponsive to CA but robustly induced by TCDD (Harper et al., 2012; Joshi et al., 2015a). Chromatin immunoprecipitation (ChIP) results confirmed that the mutually exclusive

ABBREVIATIONS: AhR, aryl hydrocarbon receptor; AhR CKO, liver-specific AhR conditional knockout; Arnt, aryl hydrocarbon receptor nuclear translocator; CA, cinnabarinic acid; ChIP, chromatin immunoprecipitation; DMSO, dimethyl sulfoxide; histone H4K5Ac, histone H4 lysine 5 acetylation; LC-MS/MS, liquid chromatography-tandem mass spectrometry; MTA2, metastatis-associated protein 2; NuRD, nucleosome remodeling and deacetylation complex; PTM, post-translational modifications; RT-PCR, reverse transcription-polymerase chain reaction; siRNA, small-interfering RNA; Stc2, stanniocalcin 2; TCDD, 2,3,7,8-tetrachlorodibenzo-p-dioxin; UTMB, University of Texas Medical Branch; XRE, xenobiotic response element. 
agonist-specific transcriptional response was dependent on AhR-DNA binding to XREs located in the regulatory regions of each gene. To interrogate the molecular basis for the agonist specificity, mass spectrometry was performed on immunoaffinity-purified AhR complexes recovered after treatment with either TCDD or CA. Metastasis tumor-associated protein 2 (MTA2) was identified as a cofactor recruited by AhR-Arnt complex exclusively in response to CA. MTA2 is a known chromatin-modifying protein, a component of nucleosome remodeling and deacetylation (NuRD) complex with the capacity to both repress and activate gene expression (Matsusue et al., 2001; Yao and Yang, 2003; Miccio et al., 2010). In the present study, we demonstrated

In the present study, we demonstrated CA-specific recruitment of MTA2-AhR complex to XREs in the Stc2 promoter with concomitant acetylation of lysine 5 on histone H4 (H4K5Ac) at the Stc2 promoter leads to transcription activation. These data identify an epigenetic mark associated with AhR activation and agonist-specific recruitment of MTA2, resulting in selective target gene induction. In addition, this study established a critical regulatory role for MTA2 in CA-dependent Stc2-mediated protection against ethanolinduced liver apoptosis.

\section{Materials and Methods}

Animals, Primary Hepatocyte Isolation, and Treatments. Eight- to ten-week-old C57BL/6 wild-type (WT), $\mathrm{AhR}^{\mathrm{fl} / \mathrm{fl}}$ (AhR-floxed), and $\mathrm{AhR}^{\mathrm{fl} / \mathrm{fl}} / \mathrm{Cre}^{\mathrm{Alb}}$ [liver-specific AhR conditional knockout (AhR $\mathrm{CKO})$ ] female mice were used in compliance with the guidelines of the Institutional Animal Care and Use Committee (IACUC) at the University of Texas Medical Branch (UTMB) at Galveston. WT, AhRfloxed, and AhR-CKO mice were treated with vehicle (peanut oil) or $20 \mu \mathrm{g} / \mathrm{kg}$ TCDD (AccuStandard, New Haven, CT) by gavage, or with dimethyl sulfoxide (DMSO) or $12 \mathrm{mg} / \mathrm{kg} \mathrm{CA}$ (in DMSO) (synthesized by Synthetic Organic Chemistry Core at UTMB) (Fazio et al., 2012) intraperitoneally for 2 and 24 hours. Primary hepatocytes were isolated using collagenase perfusion method as described previously (Harper et al., 2012). Cells were plated at a density of $10^{6}$ cells $/ \mathrm{cm}^{2}$ in Williams' E medium containing penicillin (100 IU/ml), streptomycin $(100 \mu \mathrm{g} / \mathrm{ml})$, and $5 \%$ fetal bovine serum, and treated with DMSO, $6 \mathrm{nM}$ TCDD, or $30 \mu \mathrm{M}$ CA dissolved in DMSO for 24 hours. For MTA2 knockdown studies, primary hepatocytes were transiently transfected with ON-TARGETplus MTA2 small-interfering RNA (siRNA; Thermo Fischer Scientific, Waltham, MA) for 24 hours. Metafectene PRO (Biontex Laboratories GmbH, München, Germany) was used as a transfection reagent.

Immunoaffinity Purification and Liquid ChromatographyTandem Mass Spectrometry of AhR Complexes. Liver nuclear extracts were prepared from C57BL/6 mice using a sucrose cushion as described previously (Wilson et al., 2013). Anti-AhR (Enzo Life Sciences, Farmingdale, NY, or Abcam, Cambridge, MA) and antiIgG (Cell Signaling Technology, Danvers, MA) antibodies were conjugated to M-270 epoxy Dynabeads (Thermo Fischer Scientific) per manufacturer's instructions. Antibody-conjugated magnetic beads were equilibrated with TGH buffer [50 mM HEPES, pH 7.4; $150 \mathrm{mM}$ $\mathrm{NaCl} ; 10 \%$ glycerol; $1.5 \mathrm{mM} \mathrm{MgCl} 2 ; 1 \mathrm{mM}$ EGTA; $1 \%$ Triton X-100; $1 \mathrm{mM}$ phenylmethylsulfonyl fluoride; $10 \mathrm{mM} \mathrm{NaF} ; 1 \mathrm{mM} \mathrm{Na} \mathrm{VO}_{4}$; $5 \mu \mathrm{l} / \mathrm{ml}$ protease inhibitor cocktail; and $1 \mu \mathrm{g} / \mathrm{ml}$ bovine serum albumin] and incubated with nuclear extracts from vehicle, TCDD-treated, and CA-treated C57BL/ 6 mice at $4^{\circ} \mathrm{C}$ for 6 hours. Beads were washed six times in 10 volumes TGH buffer, and the AhR complexes eluted using freshly prepared $500 \mu \mathrm{l}$ of aqueous $0.5 \mathrm{~N} \mathrm{NH}_{4} \mathrm{OH}, 0.5 \mathrm{mM}$ EDTA solution. The eluent was dried, resuspended in SDS-PAGE sample buffer, and fractionated using 4-20\% gels (Bio-Rad, Hercules, CA).
Protein bands were visualized with Coomassie for recovery as 1-mm gel slices. Protein was digested within the gel using sequencing-grade modified trypsin (Promega, Madison, WI). Liquid chromatographytandem mass spectrometry (LC-MS/MS) was performed in the Mass Spectrometry Core at the University of Texas Medical Branch using nano-LC on an LTQ Velos Orbitrap (Thermo Fischer Scientific) fitted with an electrospray ionization (ESI) source in positive-ion mode. For the LC elution gradient, $0.1 \%$ formic acid in water was used as mobile phase $\mathrm{A}$ and $0.1 \%$ formic acid in acetonitrile was used as mobile phase B. The mass resolution of the Orbitrap was set at 120,000. The ESI voltage was $2.2 \mathrm{kV}$, and the collision energy for the MS/MS experiments was $35 \mathrm{~V}$. The capillary temperature was set at $275^{\circ} \mathrm{C}$. Histone $\mathrm{H} 4$ post-translational modifications (PTMs) were determined using samples immunoprecipitated with an anti-H4 antibody (Abcam) prior to processing for LC-MS/MS. MS data files were processed using Proteome Discoverer and searched against the Uniprot-Mouse database using MASCOT (Matrix Sciences, Boston, MA). Post-translational modifications were identified using MASCOT by querying lysine acetylation as variable modification. For identification of specific lysine PTMs, MS/MS experiments were performed using the linear ion trap of the mass spectrometer at normal scan rate. All experiments were reproduced thrice.

RNA Isolation and Quantitative Reverse TranscriptionPolymerase Chain Reaction. Total RNA was isolated from primary hepatocytes using TRIzol (Life Technologies/Thermo Fisher Scientific, Carlsbad, CA) according to manufacturer's directions. First-strand cDNA was prepared from $1 \mu \mathrm{g}$ total RNA using an oligo(dT) primer (New England Biolabs, Ipswich, MA) and SuperScript II Reverse Transcriptase (Life Technologies/Thermo Fisher Scientific). Quantitative reverse transcription-polymerase chain reaction (RT-PCR) was performed in the Molecular Genomics Core facility at the University of Texas Medical Branch using ABI 7500 Fast Real-Time PCR System (Thermo Fischer Scientific).

Western Blotting and Coimmunoprecipitation. Nuclear extracts were fractionated by SDS-PAGE, transferred to Amersham Hybond-P PVDF membranes (GE Healthcare Life Sciences, Pittsburgh, PA) and probed with a rabbit polyclonal anti-AhR antibody (Enzo Life Sciences, Farmingdale, NY), goat polyclonal anti-MTA2 antibody (Santa Cruz Biotechnology Inc., Dallas, TX), histone H4 antibody (Abcam, Cambridge, MA), histone H4 lysine 5 acetylated (histone

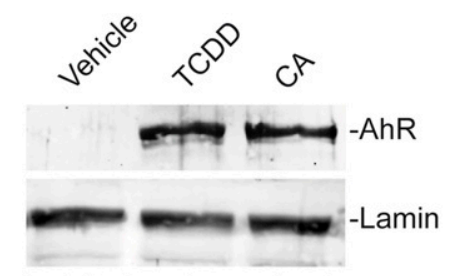

Isolated nuclear extracts

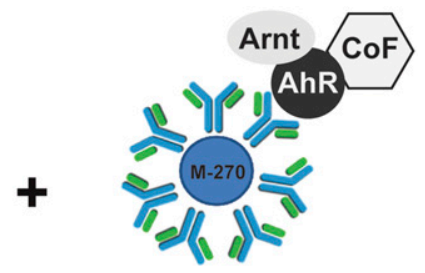

Epoxy M-270 beads coated with anti-AhR antibody
Fractionate protein by SDS-PAGE

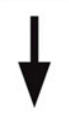

Process proteins for LC/MS-MS to identify immunopurified peptides

Fig. 1. Immunoaffinity purification with anti-AhR antibody followed by nano-LC-MS/MS to identify agonist-specific cofactors bound to AhR. Liver nuclear extracts from vehicle-, TCDD- (20 $\mu \mathrm{g} / \mathrm{kg}$ for 2 hours), and CA(12 mg/kg for 24 hours) treated C57BL6 mice were subjected to immunoprecipitation with epoxy M-270 beads coated with two separate commercially available anti-AhR antibodies (Enzo Life Sciences and Abcam). Samples were separated on 4\%-20\% Tris-HCl gels, bands excised, reduced, alkylated, trypsin-digested, and analyzed by LC-MS/MS. 

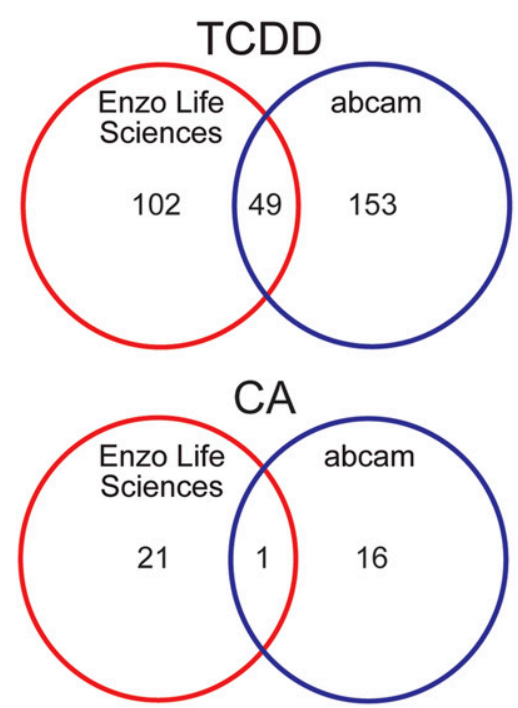

Fig. 2. Venn diagram depicting immunoprecipitated AhR-bound cofactors upon TCDD and CA treatments. Immunoprecipitation was carried out using Enzo Life Sciences and Abcam anti-AhR antibodies.

H4K5Ac) (Active Motif, Carlsbad, CA). Proteins were detected using fluorescent secondary antibodies (GE Healthcare Life Sciences) followed by imaging using a Typhoon Trio Variable Mode Imager (GE Healthcare Life Sciences). For coimmunoprecipitations, nuclear extracts $(1 \mathrm{mg})$ were equilibrated with TGH buffer and incubated with $2 \mu \mathrm{g}$ anti-MTA2 antibody (Santa Cruz Biotechnology) for 16 hours at $4^{\circ} \mathrm{C}$. Immunoprecipitations were carried out using protein A/G PLUS-Agarose beads (Santa Cruz Biotechnology), and samples analyzed using Western blotting.

Chromatin Immunoprecipitation. ChIP assays were performed on whole livers from AhR-floxed and AhR-CKO mice treated with vehicle, TCDD ( $20 \mu \mathrm{g} / \mathrm{kg})$, and CA (12 mg/kg) for 2 hours, or on primary hepatocytes isolated from C57BL/6 mice treated for 2 hours with vehicle (DMSO), $6 \mathrm{nM}$ TCDD, and $30 \mu \mathrm{M} \mathrm{CA}$, as described previously (Joshi et al., 2015a). Briefly, following treatment, liver tissues from AhR-floxed and AhR-CKO mice were finely minced and crosslinked with $1 \%$ formaldehyde in phosphate-buffered saline at room temperature for 10 minutes. Samples were homogenized using a Dounce homogenizer and centrifuged at $3200 \mathrm{~g}$ for 5 minutes at $4^{\circ} \mathrm{C}$. Pellets were resuspended in $2 \mathrm{ml}$ of cell lysis buffer ( $5 \mathrm{mM}$ PIPES, $\mathrm{pH}$ $8,85 \mathrm{mM} \mathrm{KCl}, 0.5 \%$ NP40, $4 \mu \mathrm{l}$ protease inhibitor cocktail). Samples were incubated on ice for 15 minutes, centrifuged at $3200 \mathrm{~g}$ for 5 minutes at $4^{\circ} \mathrm{C}$. Pellets were processed using ChIP-IT Express Enzymatic Kit (Active Motif) according to manufacturer's instructions.

IP: anti-MTA2 antibody

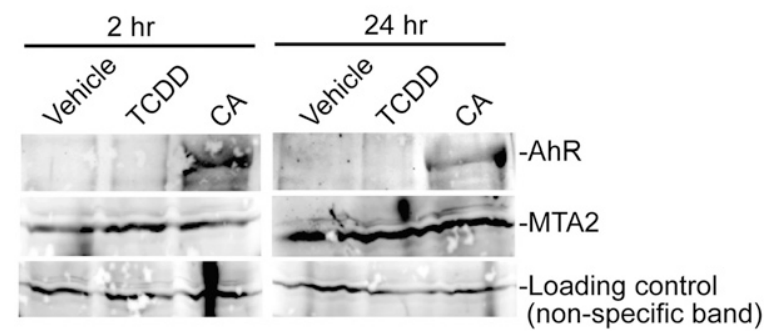

Fig. 3. Protein-protein interaction between AhR and MTA2. C57BL/6 mice were gavaged with vehicle, TCDD $(20 \mu \mathrm{g} / \mathrm{kg})$, or injected intraperitoneally with CA $(12 \mathrm{mg} / \mathrm{kg})$ for 2 and 24 hours before sacrifice. Whole liver nuclear extracts were prepared as described in Materials and Methods. Nuclear proteins $(1 \mathrm{mg})$ were immunoprecipitated with antibody against MTA2 $(2 \mu \mathrm{g})$ and immunoblotted to detect the presence of AhR.
Primary hepatocytes were crosslinked using $1 \%$ formaldehyde in Williams' E medium and processed in accordance with the manufacturer's protocol (Active Motif). Enzyme-shearing cocktail (Active Motif) was used to shear the nuclei at $37^{\circ} \mathrm{C}$ for 15 minutes. Antibodies against the AhR (Abcam), MTA2 (Santa Cruz Biotechnology), H4K5Ac (Active Motif), H4 and H3 (positive control) (Abcam), and IgG (negative control) (Cell Signaling Technology) were used to immunoprecipitate the target proteins. Immunoprecipitated and input DNA was PCR amplified using primers specific to CYP1A1 promoter flanking XREs (between -885 and -1242 from transcription start site) and stc2 promoter (between -190 and -492 from transcription start site containing $8 \mathrm{XREs})$. The CYP1A1 and Stc2 PCR primer pairs are: 5'-CTATCTCTTAAACCCCACCCCAA-3' (forward primer) and 5'-CTAAGTATGGTGGAGGAAAGGGTG-3'; (reverse primer) and $5^{\prime}$-CTCAGTCCATTCGGCCATTGCCC-3' (forward primer), and 5'-AGGAAGCGGAGCGCCTCCGC-3' (reverse primer), respectively. PCR products were fractionated on a $5 \%$ polyacrylamide gel, stained with SYBR Green (Thermo Fischer Scientific), imaged on a Typhoon Trio, and band intensities quantified using ImageQuant (GE Healthcare Life Sciences).

Caspase-3 Assays. Caspase 3 assays on isolated primary hepatocytes were performed as described previously (Harper et al., 2012). In a fluorometric caspase-3 assay, Ac-DEVD-AFC (BD Biosciences, San Jose, CA) was used as substrate. Fluorescence resulting from the cleavage of 7-amino-4-trifluoromethylcoumarin was quantified using SPECTRAmax Gemini microplate Spectrofluorometer (Molecular Devices, Sunnyvale, CA) with a 400-nm excitation filter and 505-nm emission filter.

Statistical Analysis. All data are represented as the mean \pm S.D. Statistical analyses of data were performed by UTMB Office of Biostatistics by applying analysis of variance (ANOVA) models. Differences between the groups were considered significant only if the $p$ value was $<0.05$.

\section{Results}

CA-Specific Recruitment of MTA2 by the AhR. TCDD and CA induces AhR-DNA binding, and expression of the CYP1A1 and Stc2 genes is mutually exclusive in vivo (Harper et al., 2012; Joshi et al., 2015a). However, electrophoretic mobility shift assays (EMSA) were unable to recapitulate these agonist-specific properties in vitro (Supplemental Fig. 1). AhR-Arnt DNA binding to oligonucleotide probes harboring individual CYP1A1 and Stc2 XREs revealed consistent agonist-inducible DNA binding to each XRE site in response to both ligands. This suggested that the differential transcriptional responsiveness observed was dependent on chromatin architecture and/or distinct epigenetic states, rather than an intrinsic AhR DNA-binding property imparted by the agonists. To evaluate whether AhR cofactor recruitment is agonist-specific, we isolated TCDD- and CA-treated AhR complexes from whole mouse-liver nuclear extracts by immunoaffinity purification using two different anti-AhR antibodies targeting distinct epitopes, followed by LC-MS/MS (Fig. 1). Mice were treated with vehicle, TCDD $(20 \mu \mathrm{g} / \mathrm{kg})$ for 2 hours, and CA $(12 \mathrm{mg} / \mathrm{kg})$ for 24 hours. These conditions were empirically derived on the basis of maximal CYP1A1 and Stc2 induction following TCDD and CA treatment, respectively. LC-MS/MS identified 151 (using Enzo Life Sciences anti-AhR antibody) and 202 (using Abcam anti-AhR antibody) proteins enriched following TCDD treatment, with 49 proteins common to both antibodies (Fig. 2). CA treatment resulted in the identification of 22 and 17 proteins captured using Enzo Life Sciences and Abcam anti-AhR antibodies, respectively, with only a single protein coimmunoprecipitated by both 

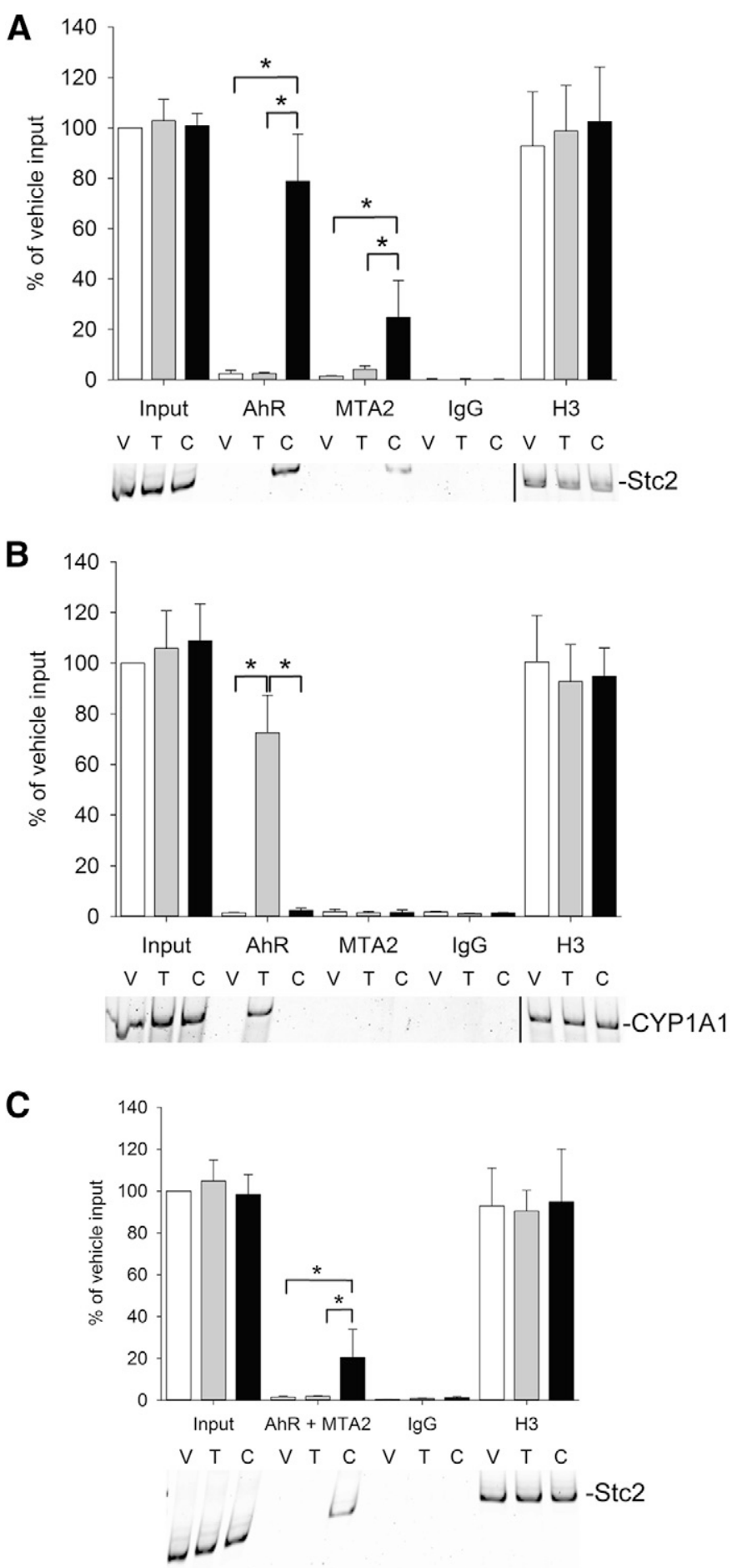

Fig. 4. CA-dependent $\mathrm{AhR}$ and MTA2 binding to the Stc2 promoter in vivo. $\mathrm{ChIP}$ assays were performed on livers from AhR-floxed mice treated with vehicle (open bars), TCDD (20 $\mu \mathrm{g} / \mathrm{kg}$ ) (gray bars), and CA (12 mg/kg) (black bars) for 2 hours. Antibodies against the AhR, MTA2, H3 (positive control), and IgG (negative control) were used to immunoprecipitate the target proteins. PCR using primers targeting XRE clusters in the Stc2 and CYP1A1 promoters were used to amplify the precipitated DNA. PCR products were loaded onto two 5\% polyacrylamide gels (represented by dividing line). Samples were ran, stained with SYBR green and imaged with Typhoon Trio imager simultaneously with exactly same image acquisition parameters. Quantification was performed using ImageQuant (GE Healthcare) software. Quantitation of PCR products against (A) Stc2 and (B) CYP1A1 promoter is presented as percentage of vehicle input DNA. (C) PCR was performed on DNA isolated in a sequential re-ChIP experiment using antibodies against the AhR followed by MTA2. For (A and B), mixed effects two-way ANOVA models were used. For (C), mixed effects one-way ANOVA antibodies. This protein was identified as MTA2, a novel component exclusively associated with the CA-activated AhR. The absence of MTA2 in the immunoprecipitates from antiIgG-probed nuclear extracts, and its absence in vehicle- or TCDD-treated complexes, is indicative of specificity for the CA-activated AhR. To verify the AhR-MTA2 interaction, coimmunoprecipitations were performed on liver nuclear extracts prepared from vehicle-, TCDD- (20 $\mu \mathrm{g} / \mathrm{kg})$, and CA(12 mg/kg) treated mice at 2 and 24 hours (Fig. 3). The data show that the AhR interacts with MTA2 only after CA treatment.

Direct Binding of AhR and MTA2 to Stc2 Promoter In Vivo in Response to CA. Previous studies established that the AhR-Arnt complex is recruited to a region of Stc2 promoter harboring a cluster of eight distinct XREs between $-193 \mathrm{bp}$ and $-450 \mathrm{bp}$ upstream of transcription start site (Harper et al., 2012; Joshi et al., 2015a). Recognizing that CA promotes an AhR-MTA2 interaction, we sought to determine if MTA2 was recruited to the XRE cluster in the Stc2 promoter. ChIP assays were performed on whole-liver tissue targeting the XREs in the Stc2 and CYP1A1 promoter regions (Fig. 4). The results reveal that both the AhR and MTA2 bind selectively to the Stc2 promoter in a CA-dependent manner (Fig. 4A). In contrast, TCDD treatment was unable to recruit AhR and MTA2 to Stc2 promoter (Fig. 4A) but, as expected, successfully recruited the AhR to the CYP1A1 promoter (Fig. 4B). Moreover, TCDD-induced AhR recruitment to the CYP1A1 promoter occurred independently of MTA2 (Fig. 4B). Re-ChIP experiments confirmed concurrent binding of the AhR and MTA2 to the Stc2 promoter, consistent with idea that both proteins are part of a DNA binding complex (Fig. 4C).

MTA2 Is Required for CA-Mediated AhR-Dependent Stc2 Gene Expression. To examine MTA2 functionality, we used RNA interference to knock down MTA2 expression in isolated murine primary hepatocytes. Western blotting confirmed that siRNAs targeting MTA2 efficiently suppressed MTA2 expression (Fig. 5A). Quantitative RT-PCR showed that loss of MTA2 expression markedly attenuated Stc2 induction in CA-treated hepatocytes but had no effect on CYP1A1 expression (Fig. 5B). These data clearly demonstrate that MTA2 is required to selectively induce Stc2 expression in response to $\mathrm{CA}$. ChIP assays demonstrated that the absence of MTA2 abolished AhR recruitment to the Stc2 promoter in CA-treated livers (Fig. 5C). Complementary studies conducted on AhR-CKO mice failed to detect MTA2 recruitment to the Stc2 promoter (Fig. 5C), indicating that DNA binding by the AhR-MTA2 complex at the Stc2 promoter is absolutely dependent on both proteins. In contrast, TCDD-inducible AhR binding to the CYP1A1 promoter occurs normally in the absence of MTA2.

CA Treatment Triggers Lysine Acetylation of Histone H4. Given that MTA2 is a component of the NuRD complex responsible for chromatin remodeling, we reasoned that MTA2 recruitment to Stc2 promoter might be associated with histone PTMs. Nuclear proteins from vehicle-, TCDD-, and CA-treated mouse livers were fractionated by SDS-PAGE and processed for

model was used. Following significant overall $F$ test from ANOVA models, the posthoc multiple comparison tests were performed for the prespecified comparisons adjusted by Bonferroni procedure. ${ }^{*} P<0.05, n=3$ independent mice. 
A

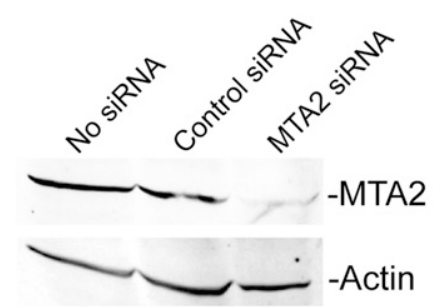

B

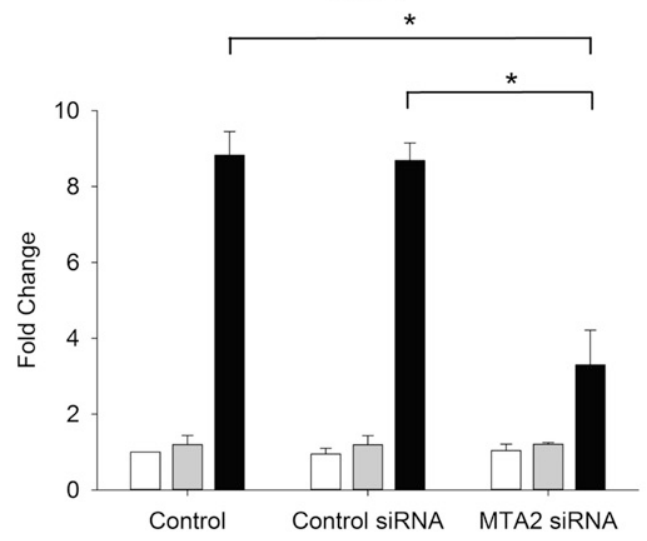

CYP1A1

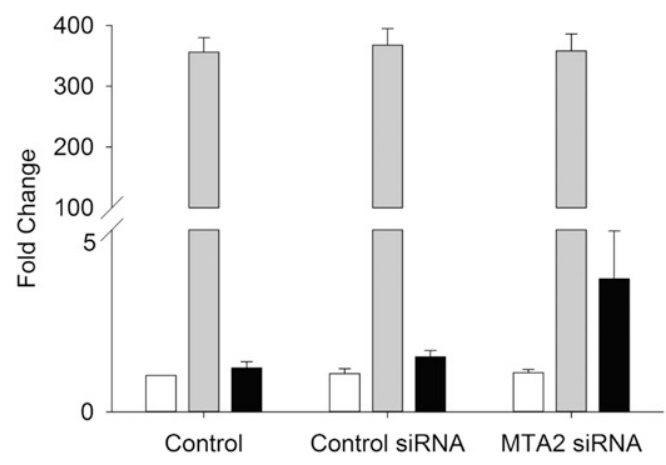

C

Stc2

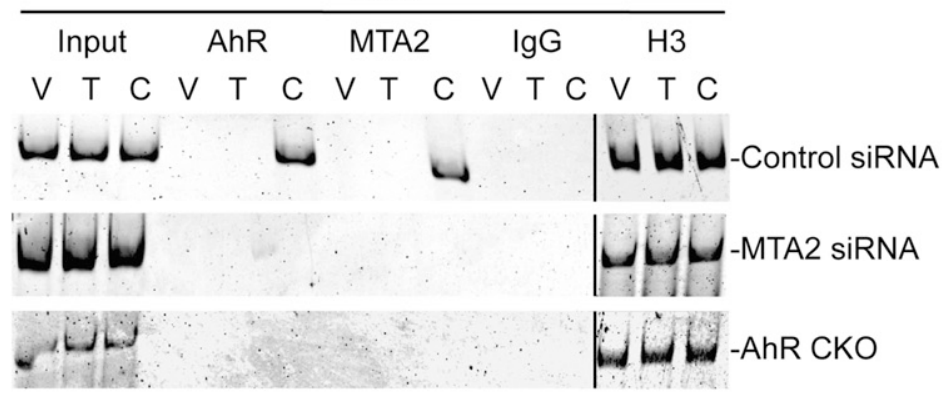

CYP1A1

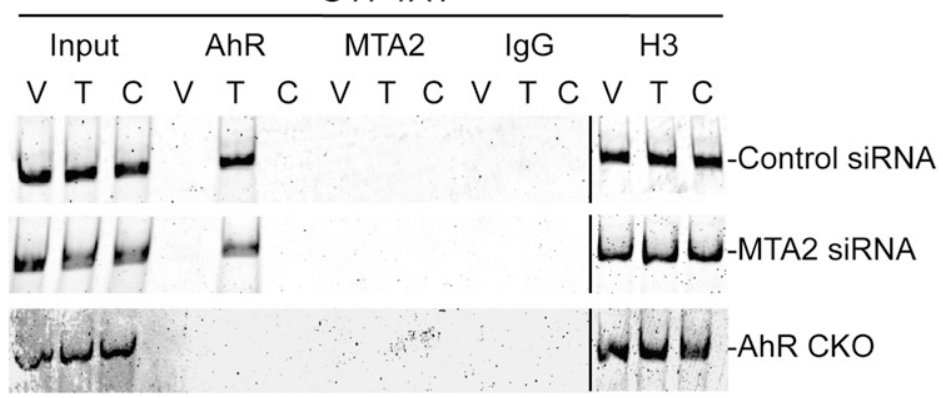

Fig. 5. MTA2 expression is required for stc2 transcriptional control. (A) Primary hepatocytes isolated from C57BL/6 mice livers were transiently transfected with an siRNA targeting MTA2 (MTA2 siRNA) or scrambled RNA (control siRNA). After 24 hours, Western blotting on total cell lysates was performed to monitor MTA2 protein expression. Actin was used as a loading control. (B) Control siRNA- and MTA2 siRNA-transfected primary hepatocytes were treated with vehicle (open bars), $6 \mathrm{nM}$ TCDD (gray bars), and $30 \mu \mathrm{M} \mathrm{CA}$ (black bars) for 24 hours. Quantitative RT-PCR was performed to detect Stc2 and CYP1A1 RNA expression and normalized to 18s rRNA. A mixed-effects multivariate ANOVA (MANOVA) model was used. Following overall significant $F$ test from MANOVA model, the posthoc multiple comparison tests were performed for the prespecified comparisons adjusted by Tukey procedure. $* P<0.05, n=$ independent batches of primary hepatocytes isolated from three mice. (C) ChIP assays performed on primary hepatocytes transiently transfected with control siRNA and MTA2 siRNA for 24 hours followed by 2-hour treatment with DMSO (V), $6 \mathrm{nM}$ TCDD (T), and $30 \mu \mathrm{M}$ CA (C). ChIP were also performed on whole-liver tissues isolated from AhR-CKO mice treated with vehicle, TCDD (20 $\mu \mathrm{g} / \mathrm{kg})(\mathrm{T})$, and CA (12 mg/kg) (C) for 2 hours. PCR products were loaded onto two $5 \%$ polyacrylamide gels (represented by dividing line), ran, stained with SYBR green and imaged on Typhoon Trio imager simultaneously with exactly same acquisition parameters. $\mathrm{n}=2$ for control siRNA and MTA2 siRNA and 1 for AhR CKO animals. 
A

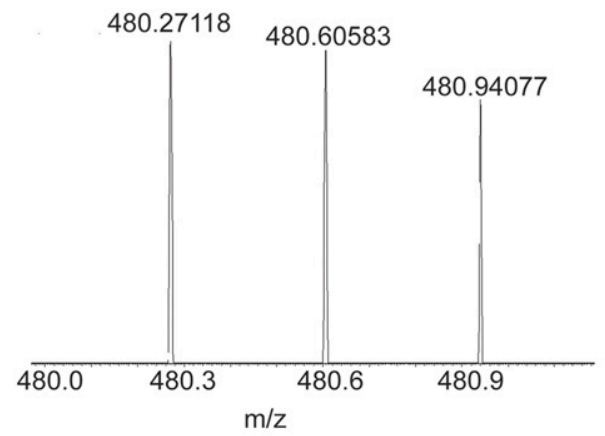

B

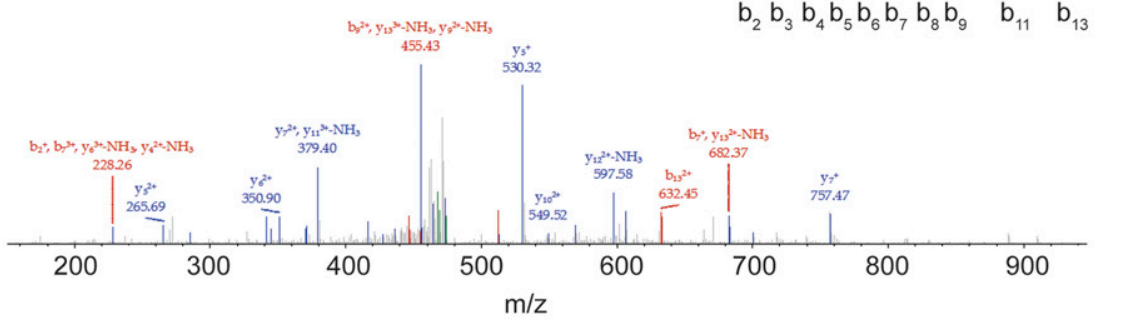

Fig. 6. Acetylation of histone $\mathrm{H} 4$ lysines exclusively upon CA treatment. (A) High-resolution MS of GkGGkGLGkGGAkR $\left[\mathrm{M}+3 \mathrm{H}^{+}\right]$. Theoretical $\mathrm{m} / \mathrm{z}=$ 480.28. Observed $\mathrm{m} / \mathrm{z}=480.28$. For unmodified peptide, $m / z=424.26$. Difference $=\sim 56=(4 \times 42) / 3$, similar to replacement of four hydrogen atoms with four acetyl groups when $z=3$. (B) High-resolution MS/MS spectra of GkGGkGLGkGGAkR $\left[\mathrm{M}+3 \mathrm{H}^{+}\right]$ encompassing residues $4-17$ in the histone $\mathrm{H} 4$. Detailed information regarding theoretical and observed $\mathrm{m} / z$ values for fragment ions are presented in (Supplemental Table 2). (C) Nuclear proteins isolated from livers of $\mathrm{C} 57 \mathrm{BL} / 6$ mice treated with vehicle, TCDD $(20 \mu \mathrm{g} / \mathrm{kg})$, and CA $(12 \mathrm{mg} / \mathrm{kg})$ for 2 and 24 hours were analyzed by Western blotting using an anti-H4K5Ac antibody $(n=3)$. Anti-histone H4 was used as a control.

C

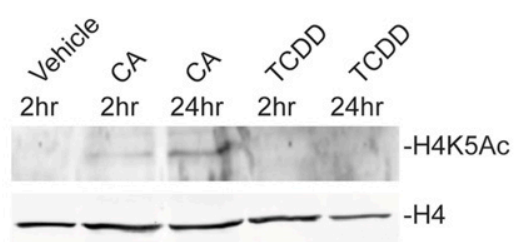

mass spectrometry to identify PTMs. In CA-treated nuclear extracts, histone $\mathrm{H} 4$ lysines in positions $5,8,12$, and to a lesser degree 16 were acetylated (Supplemental Table 1). Acetylation of histone H4 lysines was not observed in TCDD- and vehicletreated nuclear extracts (Supplemental Table 1). Furthermore CA-treated nuclear extracts immunoprecipitated with anti-H4 antibody and when subjected to MS analysis confirmed acetylation of histone $\mathrm{H} 4$ lysines 5, 8, 12, and 16 (Fig. 6, A and B). The mass of all fragment ions in the MS/MS along with the precursor ions were manually compared against the theoretical monoisotopic masses calculated using Protein
Prospector (http://prospector.ucsf.edu/). All the fragment ions that had a mass error within $2 \times$ the standard deviation of the mean error were accepted as true (Mustafa et al., 2013). Supplemental Table 2 depicts all the mass values for both precursor and fragment ions. Figure $6 \mathrm{~B}$ shows the mass accuracy and charge states for precursor peptide ion as well as MS/MS fragment ions for the peptide GkGGkGLGkGGAkR along with the sequence cleavage position leading to the formation of the ions. Using immunoblotting (Fig. 6C), we could independently confirm formation of CA-dependent acetylation of lysine 5 on histone $\mathrm{H} 4$ (H4K5Ac).
A
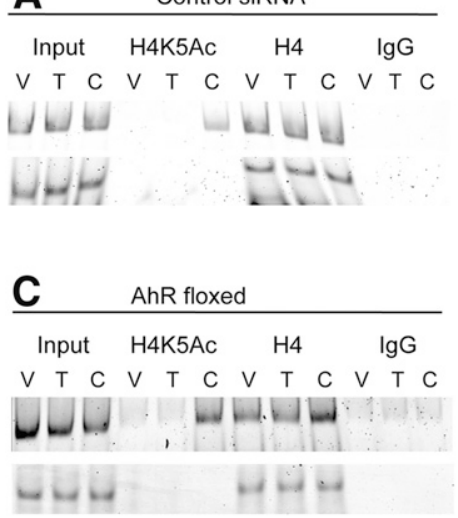
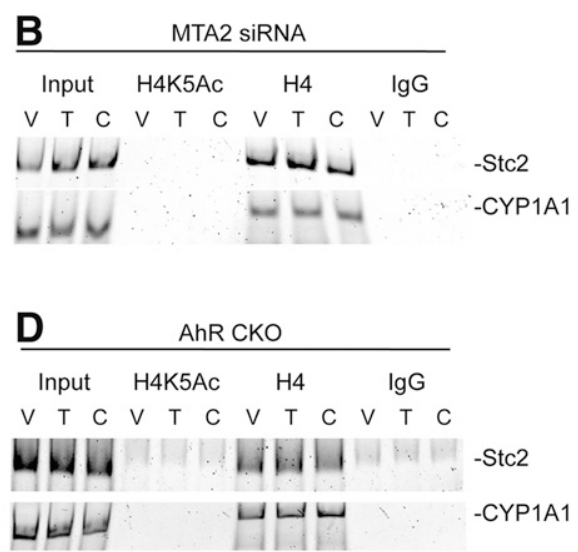

Fig. 7. Histone H4 K5 is acetylated at Stc2 promoter upon CA-dependent MTA2-AhR recruitment. Primary hepatocytes isolated from $\mathrm{C} 57 \mathrm{BL} / 6$ mice were transfected with (A) control siRNA (scrambled RNA) or (B) MTA2 siRNA (MTA2 knockdown) for 24 hours. Hepatocytes were further treated with vehicle (V), $6 \mathrm{nM}$ TCDD (T), and $30 \mu \mathrm{M} \mathrm{CA}(\mathrm{C})$ for 2 hours before ChIP. In vivo ChIP was performed using (C) AhR-floxed and (D) AhR-CKO mice treated with vehicle, TCDD $(20 \mu \mathrm{g} / \mathrm{kg})(\mathrm{T})$, and CA $(12 \mathrm{mg} / \mathrm{kg})(\mathrm{C})$ for 2 hours. Antibodies against histone H4K5Ac, H4 (positive control), and IgG (negative control) were used to immunoprecipitate target proteins. PCR primers directed against XRE cassettes in Stc2 and CYP1A1 promoters were used to amplify target DNA. PCR products were fractionated and visualized on $5 \%$ polyacrylamide gel stained with SYBR green. $n=$ 3 (for isolated primary hepatocytes) and 2 for AhR-floxed and 1 for AhR-CKO mice. 


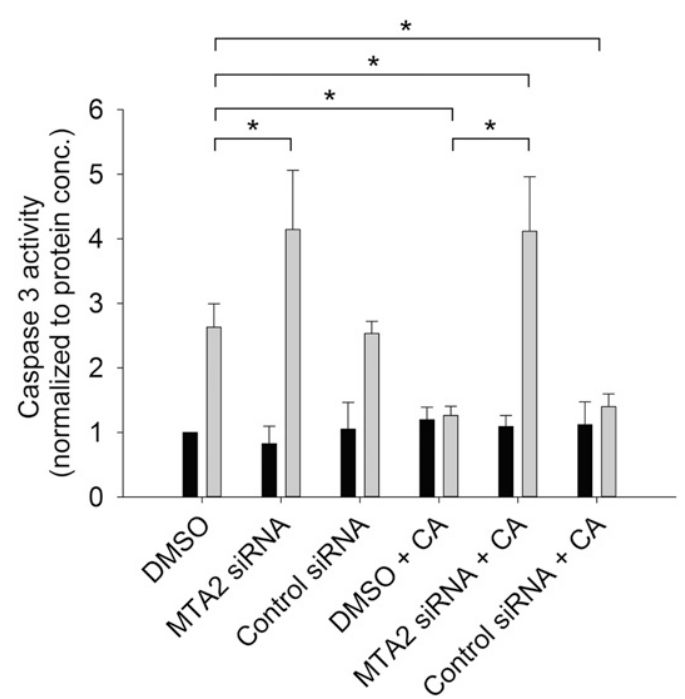

Fig. 8. MTA2 contributes to cell survival. Isolated primary hepatocytes from C57BL/6 mice were transiently transfected with control siRNA or MTA2 siRNA in the absence and presence of $30 \mu \mathrm{M}$ CA for 24 hours. Hepatocytes were subsequently treated without (black bars) or with $100 \mathrm{mM}$ ethanol (gray bars) for 24 hours. Fluorometric caspase-3 assay was performed and normalized to total protein concentration in three independent experiments. A mixed-effects two-way ANOVA model was used. Following significant overall $F$ test from the ANOVA model, the posthoc multiple comparison tests were performed for prespecified comparisons adjusted by Tukey procedure. ${ }^{*} P<0.05, n=$ independent batches of primary hepatocytes isolated from three mice.

\section{CA-Induced MTA2-AhR Recruitment to the Stc2} Promoter Is Required for Histone H4 Lysine 5 Acetylation. Murine primary hepatocytes were transiently transfected with scrambled RNA (control siRNA) or MTA2 siRNA to suppress MTA2 expression as described for Fig. 5. ChIP studies using the anti-H4K5Ac antibody revealed that CA treatment, but not TCDD treatment, led to formation of the H4K5Ac epigenetic mark in the Stc2 promoter encompassing the XRE cluster (Fig. 7A). Moreover, the H4K5Ac mark was not observed in the CYP1A1 promoter. Significantly, H4K5Ac formation was dependent on both MTA2 expression (Fig. 7B) and $\mathrm{AhR}$ expression (Fig. 7, C and D). Collectively, the data strongly suggest that transcriptional activation of the Stc2 gene by the AhR in response to CA is dependent on the selective recruitment of MTA2 - conceivably as part of the NuRD complex-resulting in epigenetic modifications, including H4K5Ac leading to chromatin changes supporting enhanced gene expression. Furthermore, these studies offer a mechanistic insight into how AhR agonists can selectively regulate gene expression.

MTA2 Is a Key Regulator of Stc2-Mediated Cytoprotection against Ethanol-Induced Apoptosis. We previously showed that CA-mediated AhR-dependent Stc2 induction was absolutely necessary to protect hepatocytes against intrinsic apoptotic cell death induced by ethanol (Joshi et al., 2015a). Given the findings reported here that MTA2 is critical for AhR-dependent Stc2 expression, we reasoned that MTA2 is likewise essential in conferring cytoprotection against an ethanol-induced injury. Murine primary hepatocytes transiently transfected with scrambled RNA (control siRNA) or MTA2 siRNA oligonucleotides were pretreated with vehicle (DMSO) or CA $(30 \mu \mathrm{M})$ for 24 hours, prior to treatment with $100 \mathrm{mM}$ ethanol for 24 hours. Apoptosis was assessed by measuring caspase-3 activity. CA conferred cytoprotection in MTA2 positive hepatocytes upon ethanol treatment but failed to protect hepatocytes with suppressed MTA2 expression (Fig. 8). This result increases our molecular understanding of endogenous AhR activity in physiologic homeostatic processes, quite distinct from the toxicological events associated with exposure to exogenous insults.

\section{Discussion}

TCDD is the prototypical AhR ligand, and studies examining CYP1A1 expression have yielded considerable insight into the basic mechanism of receptor-mediated gene expression, despite the primary biologic and toxicological effects of TCDD remaining elusive. Accumulated evidence over the last couple of decades has exposed the promiscuity with which the AhR binds a wide variety of structurally diverse ligands, triggering a myriad of toxicological and adaptive responses (Denison et al., 2011). Our recent finding that the CYP1A1 and Stc2 genes are induced mutually exclusively by TCDD and CA, respectively, despite using a common XRE-bound AhR-Arntdriven mechanism (Joshi et al., 2015a), provided an ideal model for examining the molecular basis for this response. To date, the observed variability in receptor-mediated transcriptional responses has been in part attributed to the persistence of ligands such as TCDD (Chiaro et al., 2007), ligand-specific differences in AhR XRE-dependent DNA binding (Matikainen et al., 2001), and the existence of multiple AhR DNA binding partners (Hoffman et al., 1991; Vogel et al., 2007; Wilson et al., 2013). Evidence for ligand-specific recruitment of coactivator proteins is largely absent, although Zhang et al. (2008), using a nonphysiologic mammalian two-hybrid assay, obtained data for agonist-specific coactivator interactions with the AhR, consistent with the idea that different ligands can indeed promote particular receptor-coactivator interactions. CA was recently identified as a bona fide endogenous AhR ligand (Lowe et al., 2014) that selectively induces Stc2 expression through the canonical AhR-dependent XRE-driven transcriptional response (Joshi et al., 2015a). Moreover, CA-driven Stc2 expression protects liver cells from ethanol-induced injury. The findings presented here provide the first example of physiologically relevant agonist-specific differential gene expression attributable to $\mathrm{AhR}$ recruitment of a distinct cofactor, notably MTA2, resulting in discrete epigenetic modifications on histone $\mathrm{H} 4$.

The human MTA protein family is composed of three isoforms (MTA1, MTA2, and MTA3) as well as several splice variants (Kumar and Wang, 2016). The MTA proteins do not appear to exhibit enzymatic activity nor bind DNA directly, but domain analyses suggest that the MTA proteins are capable of interacting with a large number of proteins. MTA2's role in chromatin remodeling was inferred in a proteomics study that detected MTA2 peptides in the NuRD complex (Zhang et al., 1999). The NuRD complex comprises several proteins with both ATP-dependent chromatin remodeling and histone deacetylase activities and is generally considered to be involved in transcriptional repression (Xue et al., 1998). Significantly, MTA isoforms regulate expression of target genes through NuRD-dependent as well as -independent complexes and do not coexist within the same NuRD complex. Moreover, the different MTA proteins are often functionally nonredundant (Kumar, 2014). The NuRD complex is largely regarded as a corepressor protein complex owing to the 
presence of histone deacetylases (HDACs) (Bowen et al., 2004), but the presence of HDACs per se does not ensure transcriptional repression because studies on the NuRD interaction with the GATA-1/FOG-1 transcription factor complex support transcriptional activation (Miccio et al., 2010). At this time it is unclear if AhR recruitment of MTA2 occurs in the context of a complete NuRD complex capable of coactivation as described for GATA-1/FOG-1, or if MTA2 is functioning in an NuRD-independent capacity akin to that proposed for MTA1 (Sen et al., 2014). Kim et al. (2014) recently demonstrated that two NuRD subunits, CHD4 and MTA2, associated constitutively with the DNA-bound CLOCKBMAL1 to drive target gene expression, and that transcriptional repression only occurred once the negative regulator, PERIOD, recruited the remaining NuRD complex components to CLOCK-BMAL1 DNA binding sites. Given that CLOCK and BMAL1 are both bHLH-PAS proteins belonging to the same family as the AhR and Arnt protein (Bersten et al., 2013), it is conceivable that MTA2 recruitment to the XREbound AhR is occurring in the absence of other NuRD complex components. To this end, our LC-MS/MS data did not reveal the presence of other NuRD proteins.

Both LC-MS/MS and coimmunoprecipitation studies confirmed the association of MTA2 with AhR upon CA treatment. Affinity purification using two different commercial anti-AhR antibodies followed by MS sequencing detected four unique peptides representing MTA2. Furthermore, a protein database search performed by protein BLAST (BLASTP) using four MTA2 signature peptides did not share consensus sequence with any other mouse proteins, increasing confidence in the MS data. Since MTA2 is not known to acetylate histones, it seems improbable that its recruitment to the Stc2 promoter is directly responsible for histone $\mathrm{H} 4$ acetylation. MTA2 functionality, however, is dramatically affected by p300-mediated acetylation on lysine 152 (Zhou et al., 2014). In addition, our MS finding that histone $\mathrm{H} 4$ is acetylated on $\mathrm{K} 5, \mathrm{~K} 8, \mathrm{~K} 12$, and $\mathrm{K} 16$ at the Stc2 promoter following CA treatment suggests that p300 or cAMP response element binding protein (CREB) binding protein (CBP) may be recruited to the AhR-MTA2 complex. This is consistent with the finding that both lysine acetyltransferases can acetylate these histone $\mathrm{H} 4$ residues (Henry et al., 2013), and that both p300 and CBP are recruited to the ligand-activated AhR-Arnt complex (Kobayashi et al., 1997; Hestermann and Brown, 2003). Acetylation reduces the positive charge on histones to diminish the electrostatic interactions between the histones and DNA. This leads to decondensation of the chromatin structure, thus providing access to transcription-initiation machinery (Kouzarides, 2007).

Our finding that the differential responsiveness of CYP1A1 and Stc2 to TCDD and CA, respectively, could not be recapitulated through AhR-Arnt protein DNA binding in vitro (Supplemental Fig. 1) reinforced the notion that this agonistspecific process depends on the native chromatin architecture and specific epigenetic histone modifications. The results provide compelling evidence that: 1 ) The AhR recruits MTA2 in a CA-dependent manner selectively to the Stc2 regulatory region (Fig. 4), 2) that DNA binding of the AhR-MTA2 complex is absolutely dependent on both proteins (Fig. 5), and 3) that MTA2 recruitment to the Stc2 regulatory region is necessary for histone $\mathrm{H} 4$ acetylation (Fig. 7). The LC-MS/MS results showed that AhR-MTA2 DNA binding induced histone H4 lysine acetylation at positions K5, K8, K12, and K16. Acetylation of $\mathrm{H} 4 \mathrm{~K} 5$ was independently verified using immunologic methods. Western blotting using the anti-H4K5Ac antibody indicated that maximal histone $\mathrm{H} 4$ acetylation occurred at 24 hours in CA-treated nuclear extracts (Fig. 6B), which correlates closely with kinetics for Stc2 mRNA accumulation reported previously (Joshi et al., 2015a), suggesting that this histone mark is important in Stc2 gene expression. Precisely how H4K5 (and the other lysines) becomes acetylated, the role of MTA2 in epigenetic changes at other AhR target genes, and the nature of the AhR-MTA2 interaction will be examined in future studies.

Collectively, these observations attest to a mechanism whereby AhR agonists can selectively affect the epigenetic profile of XRE-driven target genes to influence the transcriptional response. This process is distinct from the recently described AhR-mediated epigenetic changes associated with receptor binding at nonconsensus XREs and concomitant recruitment of CPS1, resulting in histone $\mathrm{H} 1$ homocitrullination (Joshi et al., 2015b). The studies indicate that a complete understanding of AhR transcriptional regulation will require a more thorough examination of the epigenetic process associated with AhR activity in response to various agonists.

\section{Acknowledgments}

The authors thank Dr. Larry Denner for helpful discussions regarding MS data analysis and Dr. Xiaying Yu, Office of Biostatistics at UTMB, for statistical support. Samples were analyzed at the Mass Spectrometry Core of the Biomolecular Resource Facility and at the Molecular Genomics Core in the University of Texas Medical Branch.

\section{Authorship Contributions}

Participated in research design: Joshi, Elferink.

Conducted experiments: Joshi.

Contributed new reagent or analytical tools: Joshi, Hossain, Elferink.

Performed data analysis: Joshi, Hossain, Elferink.

Wrote or contributed to the writing of the manuscript: Joshi, Hossain, Elferink.

\section{References}

Beischlag TV, Luis Morales J, Hollingshead BD, and Perdue GH (2008) The aryl hydrocarbon receptor complex and the control of gene expression. Crit Rev Eukaryot Gene Expr 18:207-250.

Bersten DC, Sullivan AE, Peet DJ, and Whitelaw ML (2013) bHLH-PAS proteins in cancer. Nat Rev Cancer 13:827-841.

Bowen NJ, Fujita N, Kajita M, and Wade PA (2004) Mi-2/NuRD: multiple complexes for many purposes. Biochim Biophys Acta 1677:52-57.

Chiaro CR, Patel RD, Marcus CB, and Perdew GH (2007) Evidence for an aryl hydrocarbon receptor-mediated cytochrome p450 autoregulatory pathway. Mol Pharmacol 72:1369-1379.

Denison MS, Soshilov AA, He G, DeGroot DE, and Zhao B (2011) Exactly the same but different: promiscuity and diversity in the molecular mechanisms of action of the aryl hydrocarbon (dioxin) receptor. Toxicol Sci 124:1-22.

Fazio F, Lionetto L, Molinaro G, Bertrand HO, Acher F, Ngomba RT, Notartomaso S, Curini M, Rosati O, Scarselli P, et al. (2012) Cinnabarinic acid, an endogenous metabolite of the kynurenine pathway, activates type 4 metabotropic glutamate receptors. Mol Pharmacol 81:643-656.

Goodale BC, Tilton SC, Corvi MM, Wilson GR, Janszen DB, Anderson KA, Waters KM, and Tanguay RL (2013) Structurally distinct polycyclic aromatic hydrocarbons induce differential transcriptional responses in developing zebrafish. Toxicol Appl Pharmacol 272:656-670.

Harper TA, Jr, Joshi AD, and Elferink CJ (2012) Identification of stanniocalcin 2 as a novel aryl hydrocarbon receptor target gene. J Pharmacol Exp Ther 344:579-588. Henry RA, Kuo YM, and Andrews AJ (2013) Differences in specificity and selectivity between CBP and p300 acetylation of histone H3 and H3/H4. Biochemistry 52: $5746-5759$

Hestermann EV and Brown M (2003) Agonist and chemopreventative ligands induce differential transcriptional cofactor recruitment by aryl hydrocarbon receptor. $\mathrm{Mol}$ Cell Biol 23:7920-7925.

Hoffman EC, Reyes H, Chu FF, Sander F, Conley LH, Brooks BA, and Hankinson O (1991) Cloning of a factor required for activity of the Ah (dioxin) receptor. Science 252:954-958. 
Hrubá E, Vondráček J, Líbalová H, Topinka J, Bryja V, Souček K, and Machala M (2011) Gene expression changes in human prostate carcinoma cells exposed to genotoxic and nongenotoxic aryl hydrocarbon receptor ligands. Toxicol Lett 206 $178-188$.

Joshi AD, Carter DE, Harper TA, Jr, and Elferink CJ (2015a) Aryl hydrocarbon receptor-dependent stanniocalcin 2 induction by cinnabarinic acid provides cytoprotection against endoplasmic reticulum and oxidative stress. $J$ Pharmacol Exp Ther 353:201-212.

Joshi AD, Mustafa MG, Lichti CF, and Elferink CJ (2015b) Homocitrullination is a novel histone $\mathrm{H} 1$ epigenetic mark dependent on aryl hydrocarbon receptor recruitment of carbamoyl phosphate synthase 1. J Biol Chem 290:27767-27778.

Kim JY, Kwak PB, and Weitz CJ (2014) Specificity in circadian clock feedback from targeted reconstitution of the NuRD corepressor. Mol Cell 56:738-748.

Kobayashi A, Numayama-Tsuruta K, Sogawa K, and Fujii-Kuriyama Y (1997) CBP/ p300 functions as a possible transcriptional coactivator of Ah receptor nuclear translocator (Arnt). $J$ Biochem 122:703-710.

Kouzarides T (2007) Chromatin modifications and their function. Cell 128:693-705.

Kumar R (2014) Functions and clinical relevance of MTA proteins in human cancer. Preface. Cancer Metastasis Rev 33:835.

Kumar R and Wang RA (2016) Structure, expression and functions of MTA genes. Gene 582:112-121.

Lowe MM, Mold JE, Kanwar B, Huang Y, Louie A, Pollastri MP, Wang C, Patel G, Franks DG, Schlezinger J, et al. (2014) Identification of cinnabarinic acid as a novel endogenous aryl hydrocarbon receptor ligand that drives IL-22 production. PLoS One 9:e87877.

Matikainen T, Perez GI, Jurisicova A, Pru JK, Schlezinger JJ, Ryu HY, Laine J, Sakai T, Korsmeyer SJ, Casper RF, et al. (2001) Aromatic hydrocarbon receptordriven Bax gene expression is required for premature ovarian failure caused by biohazardous environmental chemicals. Nat Genet 28:355-360.

Matsusue K, Takiguchi S, Toh Y, and Kono A (2001) Characterization of mouse metastasis-associated gene 2: genomic structure, nuclear localization signal, and alternative potentials as transcriptional activator and repressor. DNA Cell Biol $\mathbf{2 0}$ 603-611.

Miccio A, Wang Y, Hong W, Gregory GD, Wang H, Yu X, Choi JK, Shelat S, Tong W, Poncz M, et al (2010) NuRD mediates activating and repressive functions of GATA-1 and FOG-1 during blood development. EMBO J 29:442-456.

Mustafa MG, Petersen JR, Ju H, Cicalese L, Snyder N, Haidacher SJ, Denner L, and Elferink C (2013) Biomarker discovery for early detection of hepatocellular carcinoma in hepatitis C-infected patients. Mol Cell Proteomics 12 3640-3652.

Opitz CA, Litzenburger UM, Sahm F, Ott M, Tritschler I, Trump S, Schumacher T, Jestaedt L, Schrenk D, Weller M, et al. (2011) An endogenous tumour-promoting ligand of the human aryl hydrocarbon receptor. Nature 478:197-203.
Ovando BJ, Ellison CA, Vezina CM, and Olson JR (2010) Toxicogenomic analysis of exposure to TCDD, PCB126 and PCB153: identification of genomic biomarkers of exposure to AhR ligands. BMC Genomics 11:583.

Rannug U, Rannug A, Sjöberg U, Li H, Westerholm R, and Bergman J (1995) Structure elucidation of two tryptophan-derived, high affinity Ah receptor ligands. Chem Biol 2:841-845.

Sen N, Gui B, and Kumar R (2014) Physiological functions of MTA family of proteins. Cancer Metastasis Rev 33:869-877.

Soshilov AA and Denison MS (2014) Ligand promiscuity of aryl hydrocarbon receptor agonists and antagonists revealed by site-directed mutagenesis. Mol Cell Biol 34: 1707-1719.

Vogel CF, Sciullo E, Li W, Wong P, Lazennec G, and Matsumura F (2007) RelB, a new partner of aryl hydrocarbon receptor-mediated transcription. Mol Endocrinol 21: 2941-2955.

Wei YD, Helleberg H, Rannug U, and Rannug A (1998) Rapid and transient induction of CYP1A1 gene expression in human cells by the tryptophan photoproduct 6-formylindolo[3,2-b]carbazole. Chem Biol Interact 110:39-55.

White SS and Birnbaum LS (2009) An overview of the effects of dioxins and dioxinlike compounds on vertebrates, as documented in human and ecological epidemiology. J Environ Sci Health C Environ Carcinog Ecotoxicol Rev 27:197-211.

Wilson SR, Joshi AD, and Elferink CJ (2013) The tumor suppressor Kruppel-like factor 6 is a novel aryl hydrocarbon receptor DNA binding partner. J Pharmacol Exp Ther 345:419-429.

Xue Y, Wong J, Moreno GT, Young MK, Côté J, and Wang W (1998) NURD, a nove complex with both ATP-dependent chromatin-remodeling and histone deacetylase activities. Mol Cell 2:851-861.

Yao YL and Yang WM (2003) The metastasis-associated proteins 1 and 2 form distinct protein complexes with histone deacetylase activity. J Biol Chem 278:42560-42568

Zhang S, Rowlands C, and Safe S (2008) Ligand-dependent interactions of the Ah receptor with coactivators in a mammalian two-hybrid assay. Toxicol Appl Pharmacol 227:196-206.

Zhang Y, Ng HH, Erdjument-Bromage H, Tempst P, Bird A, and Reinberg D (1999) Analysis of the NuRD subunits reveals a histone deacetylase core complex and a connection with DNA methylation. Genes Dev 13:1924-1935.

Zhou J, Zhan S, Tan W, Cheng R, Gong H, and Zhu Q (2014) P300 binds to and acetylates MTA2 to promote colorectal cancer cells growth. Biochem Biophys Res Commun 444:387-390.

Address correspondence to: Dr. Aditya D. Joshi, Department of Pharmacology and Toxicology, University of Texas Medical Branch, 301 University Boulevard, Galveston, TX 77555-0654. E-mail: adjoshi@utmb.edu 